\title{
Lumen
}

Selected Proceedings from the Canadian Society for Eighteenth-Century Studies

\section{Les infortunes des Oeuvres de Jean Racine (Pierre Didot, 1801-1805) : réflexions sur la production et la réception d'un livre-monument}

\section{Annie Champagne}

Volume 36, 2017

URI : https://id.erudit.org/iderudit/1037855ar

DOI : https://doi.org/10.7202/1037855ar

Aller au sommaire du numéro

Éditeur(s)

Canadian Society for Eighteenth-Century Studies / Société canadienne d'étude du dix-huitième siècle

ISSN

1209-3696 (imprimé)

1927-8284 (numérique)

Découvrir la revue

Citer cet article

Champagne, A. (2017). Les infortunes des Oeuvres de Jean Racine (Pierre Didot, 1801-1805) : réflexions sur la production et la réception d'un livre-monument. Lumen, 36, 75-94. https://doi.org/10.7202/1037855ar 


\title{
Les infortunes des (Euvres de Jean Racine (Pierre Didot, 1801-1805) : réflexions sur la production et la réception d'un livre-monument
}

\author{
Annie Champagne \\ Université du Québec à Montréal
}

Du théâtre français l'honneur et la merveille, Il sut ressusciter Sophocle en ses écrits, Et dans l'art d'enchanter les cœurs et les esprits Surpasser Euripide, et balancer Corneille ${ }^{1}$.

Par ces vers, l'éditeur-imprimeur Pierre Didot (1760-1853) célèbre Jean Racine (1639-1699) et son théâtre, qu'il estime incarner la quintessence de la tradition littéraire française. Tiré du prospectus de 1799, dans lequel Didot annonce son édition de grand luxe des pièces de Racine, cet extrait révèle les sources antiques privilégiées par le tragédien de même qu'il insinue le débat au long cours, relancé pendant plus de deux siècles, entre Racine et Corneille. En filigrane de l'éloge qui s'impose d'emblée dans ces vers, il faut y voir se dessiner le climat culturel du tournant de 1800 teinté par l'essor des nationalismes modernes. En quête de son identité nationale, la France révolutionnée exhibe ses racines et fait revivre ses ancêtres, elle «panthéonise» ses grands hommes sur la base toute démocratique du mérite et du talent ${ }^{2}$. Racine et son théâtre sont estimés comme des joyaux nationaux, et c'est dans ce contexte qu'il faut envisager la publication des Euvres de

1. Didot, dans le prospectus du Racine daté de 1799, cité par Carol Margot Osborne, Pierre Didot the Elder and French Book Illustration: 1789-1822, Stanford, Stanford University, Department of Art, Thèse de doctorat, juin 1979, p. 114.

2. Mona Ozouf, «Le Panthéon", Les lieux de mémoire, vol. I, La République, Paris, Gallimard, 1997, p. 156. 
Jean Racine, grand in-folio illustré en 3 volumes et publié chez Pierre Didot à Paris, à partir de $1801^{3}$. Par de telles entreprises d'envergure, l'éditeur embrasse la cause patriotique et cherche à «attester la splendeur générale du gouvernement ${ }^{4} »$.

Le contexte de production du Racine révèle une triple monumentalité - typographique, littéraire et politique - qui constitue le pilier de la stratégie éditoriale de Pierre Didot. Conçu comme un hommage, célébrant la mémoire et l'œuvre du tragédien classique, l'ouvrage honore du même souffle le nouveau régime consulaire (1799-1804) et souligne l'excellence des artisans français du livre. En dépit d'une stratégie éditoriale ambitieuse et, pouvait-on penser, infaillible, le plus prestigieux livre de Pierre Didot n'a suscité l'enthousiasme ni des commentateurs ni des collectionneurs de son temps. À la critique trop peu loquace se greffe la déroute commerciale de l'entreprise qui s'est avérée un gouffre financier. Ces «infortunes » marquant la réception du Racine contrastent avec l'exubérance de sa production pour laquelle Didot déploie la grande pompe éditoriale.

Pour mieux cerner la signification et la valeur culturelles des Euvres de Jean Racine, je m'intéresse dans ces pages à l'édification du livre-monument et à sa triple monumentalité, puis à sa réception mitigée. Comment interpréter ces infortunes à l'aune de la tradition des livres à gravures, de la valeur culturelle du théâtre racinien et du contexte politique et culturel dans lequel l'édition est reçue? Je propose en conclusion quelques pistes de réflexion pour mieux comprendre l'accueil formel qu'on lui réserve, sans tambour ni trompette, malgré la perfection présumée de sa matérialité et la vitalité du culte racinien au tournant du siècle.

3. Pierre Didot l'Aîné, (Euvres de Jean Racine, Paris, Imprimerie de P. Didot l’Aîné au Palais National des Sciences et des Arts, 1801-1805, 3 volumes. Louvrage est annoncé dans le Journal typographique et bibliographique du 8 (avec prospectus) et du 18 août 1801 .

4. Didot, dans une lettre de 1804, cité par Carol Margot Osborne, op. cit., p. 144.

5. Nombre d'historiens ont eu recours à cette analogie entre le livre et le monument pour la description des (Euvres de Jean Racine. L'un des plus éloquents, François Barbier, affirme que «le format du Racine de 1801 en fait, selon le désir même de l'imprimeur, un véritable monument, que sa présentation rapproche d'un programme architectural ». Voir François Barbier et David Bellos, «Le livre au temps des Didot», dans Henri-Jean Martin et Roger Chartier (éd.), Le livre triomphant: 1660-1830, Paris, Promodis, 1984, p. 574. 


\section{Le monument typographique}

Sous la Révolution et jusque dans les premières années de l'Empire, Pierre Didot est estimé comme l'un des membres les plus marquants de la célèbre dynastie d'imprimeurs français, par l'ennoblissement du livre qu'il a accompli. La série dite «du Louvre», conçue au Palais National des Sciences et des Arts d'où elle tire son nom, constitue l'apogée de l'esthétique Didot, habile conjugaison de grandiose et de dépouillement. Cette série a été publiée à partir de 1798, après que l'éditeur-imprimeur ait eu le privilège d'installer ses presses au Louvre, «à titre d'artiste», insigne honneur réservé aux grands créateurs. L'édition des Bucoliques, des Géorgiques et de l'Énéide de Virgile (1798) est le premier livre de peintre ${ }^{6}$ d'un ensemble de cinq qui installe les particularités du genre, peaufinées dans l'édition des Odes d'Horace de 1799 puis, plus encore, dans les Euvres de Jean Racine de 1801. Suivront les Fables de La Fontaine en 1802 et, beaucoup plus tardivement en 1819, les Euvres de Boileau.

Dans la préface qui ouvre le premier volume du Racine, Didot explique son ambition :

$\mathrm{Si}$, au milieu des secousses inséparables d'une révolution, et toujours affligeantes pour les arts, j’ai pu amener à une heureuse fin l'ensemble de cette édition remarquable, il ne m’a fallu rien de moins qu'une constance inaltérable et un désintéressement total, joints au désir ardent d'élever à la gloire de Racine un monument typographique qui devint pour ainsi dire national'7.

En qualité d'éditeur-imprimeur, Didot s’approprie la métaphore monumentale et conçoit un monument matériel qui se décline en trois imposants volumes in-folio ${ }^{8}$. Cette analogie entre le livre et l'architecture est renforcée dans le Racine par les matériaux de première qualité choisis par l'éditeur : reliure de maroquin finement ornée, papier vélin

6. Livre de peintre, livre à gravures, livre à figures, autant d'appellations qui conviennent aux éditions du Louvre, précédant l'ère du livre illustré et de la vignette caractéristiques du XIX ${ }^{\mathrm{e}}$ siècle. Dans le prospectus (1797) annonçant le Virgile, Didot prévient d'ailleurs que les figures de ses éditions « toutes très soignées, ne ressemblent en rien aux vignettes que l'on voit communément dans les livres ».

7. Pierre Didot l'Aîné, «L'imprimeur au lecteur », Euvres de Jean Racine, op. cit., t. I, page 2.

8. La copie 105 de 250 consultée à l'Université de Montréal mesure près de 50 par $35 \mathrm{~cm}$. 
de la prestigieuse fabrique de Joseph de Montgolfier d'Annonay et encre la plus noire qu'il ait été possible de développer'. La mise en page géométrique et sobre accentue l'impression de majesté qui se dégage de l'ouvrage et se trouve en parfaite harmonie avec l'angulosité des caractères perfectionnés par Firmin Didot, frère et proche collaborateur de Pierre. Lespace du livre joue donc de contrastes entre la blancheur de la page et l'élégance de la typographie dépouillée qui se refuse aux arrondis et aux fioritures, tels les culs-de-lampe, vignettes et autres enjolivures innombrables dans le livre du XvIII ${ }^{\mathrm{e}}$ siècle. La sobriété formelle touche toutes les dimensions du Racine qui rappelle en outre le livre du XVII ${ }^{\mathrm{e}}$ siècle, envisagé avec la même grandiloquence. Les folios sont alors récurrents et témoignent d'une conception du livre comme «objet social qui vise à la pérennité et à la monumentalité » contrairement aux petits formats qui sont de l'ordre de l'intime, des objets à tenir dans la main, servant l'appropriation individuelle ${ }^{10}$. Au $\mathrm{XVIII}{ }^{\mathrm{e}}$ siècle, les ouvrages en format in- $12^{\circ}$ abondent tandis que l'in-folio semble anachronique, tiré de temps anciens avec lesquels Didot renoue volontiers pour ses éditions du Louvre.

Si l'esthétique du livre se prête au parallèle monumental, il en va de même de ses usages alors que la lecture s'appréhende à la manière d'un voyage par le lecteur qui pénètre le livre et s'y trouve absorbé. Frontispice, préface, et autres pages liminaires apparaissent alors comme autant de seuils qui annoncent le programme de lecture tandis que les illustrations accompagnent le lecteur et le guident. Pour son Racine, Didot l'annonce en préface, il «offre en tableau le trait le plus intéressant de chacun des actes ${ }^{11}$ », soit cinquante-six images (cinquante-sept avec le frontispice) qui appuient certains moments cruciaux de la trame narrative des œuvres théâtrales du répertoire racinien $^{12}$.

Les illustrations ont été reproduites sur cuivre, au burin, lequel fonde la réputation de la grande gravure française depuis le XVII siècle.

9. Carol Margot Osborne, op. cit., p. 53.

10. Alain-Marie Bassy, «Le texte et l'image», dans Henri-Jean Martin et Roger Chartier (éd.), op. cit., p. 148.

11. Pierre Didot L'Aîné, op. cit., p. 1 de l'avis de l'imprimeur.

12. Onze tragédies en cinq actes sont illustrées par autant de planches, sauf la comédie Les Plaideurs et la tragédie historique Esther qui ne comptent chacune que trois actes et donc trois illustrations. 
Ce procédé rigoureux amplifie la solennité des images qui reflètent par ailleurs la passion pour l'Antiquité gréco-romaine touchant toutes les sphères de l'esthétique européenne dans la seconde moitié du XVIII ${ }^{\mathrm{e}}$ siècle. Les Didot contribuent à ce climat culturel particulier. À l'heure où les Français s'imaginent tels les héritiers de la Rome républicaine, le livre est nourri des mêmes aspirations historicistes et se pare d'attributs à l'antique. À cet égard, les illustrations du Racine exemplifient le langage formel du néoclassicisme français, pétri d'Antiquité, et largement établi par Jacques-Louis David (1748-1825) dans la peinture de la fin du XviII siècle. C'est d'ailleurs à David que Didot a confié la supervision des travaux des artistes - huit peintres et sculpteurs et vingt-cinq graveurs, pour la plupart issus de son atelier - quand le maître n'a pas lui-même soumis dessins et idées, tant pour le Racine que le Virgile et l'Horace ${ }^{13}$. Si les images méritent d'être étudiées en profondeur pour cibler les distinctions entre les «mains » qui y ont pris part $^{14}$, l'esprit général se dégageant de cet ensemble en est un de rigueur à l'antique (figures 1 et 2). Cette unité visuelle était recherchée par Didot qui souhaitait que les illustrations soient en parfaite cohésion avec la mise en livre et la typographie. Telles de petites peintures d'histoire, elles sont isolées sur une page liminaire en ouverture d'acte et cintrées d'une fine bordure s'apparentant à un cadre. Conçues comme des peintures, elles étaient estimées comme telles, non pas le fruit de «vulgaires illustrateurs ${ }^{15}$ », mais bien d'« artistes distingués [...] accoutumés déjà aux éloges mérités du public », tels que les présente Didot $^{16}$. En qualité de peintres, les Girodet, Gérard et Cie ont rehaussé le statut de l'illustration et, conséquemment, le statut même du livre.

13. David a collaboré à la réalisation de 92 planches pour ces trois éditions du Louvre. Voir Carol Margot Osborne, op. cit., p. 84.

14. Pour une analyse soutenue des illustrations du Racine, voir le récent article d'Elizabeth M. Rudy consacré à cet ensemble hétérogène exemplifiant ce que l'auteure définit comme le néoclassicisme «moderne». Elizabeth M. Rudy, «Making a Classic Modern: Pierre Didot l'aîné's illustrated Euvres de Jean Racine», Word ङ Image, 30, 2, avril-juin 2014, p. 104-116.

15. L'illustrateur professionnel a été déconsidéré jusqu'au XIX ${ }^{\mathrm{e}}$ siècle. Avant 1830, il était perçu comme une «sorte de sous-auteur » jusqu'à ce qu'il acquiert une notoriété par l'entremise des revues et de la presse. Michel Melot, «À l'ombre des images. Nouvelles approches de l'histoire de l'illustration ", Histoire de l'art, 45, décembre 1999 , p. 6.

16. Pierre Didot L'Aîné, op. cit., p. 1 de l'avis de l'imprimeur. 
Le monument typographique livresque tend à accéder à la majesté des formes architecturales, mais aussi à la dignité de la peinture d'histoire.

La stratégie éditoriale élaborée par Didot repose tout autant sur le tirage, limité à 250 exemplaires pour toutes les éditions du Louvre, que sur des finesses destinées à susciter l'émulation des collectionneurs. Par exemple, la publication d'éditions agrémentées de séries gravées avant la lettre ou encore, plus rarissimes, contenant les dessins origi$n^{n a u x}{ }^{17}$, rehaussait la valeur de l'ouvrage. En outre, chaque copie de la série du Louvre a été numérotée et signée par l'éditeur (figure 3$)^{18}$. De nature sérielle, le livre se trouve alors doté d'une aura qui en fait une pièce exclusive : d'objet de consommation, il acquiert la noblesse de l'objet de collection. Cette signature permet du reste à Didot de s'attribuer le prestige de l'ouvrage, chef-d'œuvre de son imprimerie, du Louvre et de la France. Les Euvres de Jean Racine sont d'ailleurs «dans tous ses éléments, le produit de l'industrie nationale» annonce Didot en ouverture de son édition, à laquelle il a voulu conférer "plus de magnificence encore » que les éditions du Louvre précédentes, soit celles des Latins Virgile (1798) et Horace (1799) ${ }^{19}$. Le Racine compte non seulement plus d'illustrations que toutes les éditions de la série réunies $(57$ grandes planches contre 23 pour le Virgile, 5 pour les Fables de La Fontaine et 12 bandeaux pour l'Horace, 9 pour le Boileau), mais celles-ci sont plus majestueuses que celles du Virgile et des Fables et, conséquemment, les caractères, plus forts. À cette époque de conscience nationale exacerbée, le Racine est envisagé comme une démonstration du génie français pour laquelle Didot, par sa signature manuscrite, pérennise son rôle de maître d'œuvre. Le monument

17. Les dessins originaux du Racine et du Virgile ont été conservés dans un exemplaire unique que Pierre Didot a offert à son frère Firmin. En 1824, le précieux livre est entré à la bibliothèque nationale de France sans les dessins qui auraient été dispersés. C'est le constat que tirent la plupart des historiens, notamment A. J. Pons, Les éditions illustrées de Racine, Paris, A. Quantin, 1878, p. 39. Pour consulter la plus récente liste des dessins du Racine conservés dans les collections publiques, voir Marie-Claire Planche, De l'iconographie racinienne, dessiner et peindre les passions, Turnhout, Brepols, 2010, p. 92-96.

18. Cette tendance - le numérotage et la signature - était alors nouvelle et récurrente dans les années 1790, en France. Anthony Griffiths, Prints for Books: Book Illustration in France, 1760-180o, Londres, British Library, 2000, p. 143.

19. Pierre Didot L'Aîné, op. cit., p. 2 de l'avis de l'imprimeur. Deuxième extrait tiré du prospectus du Virgile (1797) et cité par Osborne, op. cit., p. 131. 
typographique en vient à égaler le monument littéraire que constitue le théâtre de Racine.

\section{Le monument littéraire}

Par son édition de 1801, Pierre Didot célèbre la valeur historique du théâtre de Racine, monument littéraire qu'il fait bon de rappeler à la mémoire collective nationale. Jusque dans les années 1830, le théâtre de Racine est d'ailleurs estimé comme un modèle spécifiquement français dont la célébration s'apparente à un devoir patriotique ${ }^{20}$. Cette quête de l'identité nationale est à juste titre un moteur non seulement du Racine, mais de l'ensemble des éditions du Louvre qui font la part belle à la tradition française. Par ses éditions des «classiques» français, Didot espère voir rejaillir en son temps, un peu du prestige du XVII ${ }^{\text {e }}$ siècle, «le plus vanté de l'histoire nationale», bien que son culte n'ait pas fait l'unanimitée ${ }^{1}$. Les classiques du Grand siècle sont instrumentalisés en 1800, gardiens du temple pour les uns, vestiges de l'absolutisme pour les autres ${ }^{22}$. Dès le XVII ${ }^{\mathrm{e}}$ siècle cependant, le talent de Racine le canonise comme un héritier légitime des anciens, tel que le suggère d'ailleurs l'iconographie du frontispice allégorique (figure 4) de l'édition de 1801. Dans la composition, Melpomène, muse de la tragédie, et son génie ailé conduisent un Racine guilleret à la figure de l'immortalité qui le couronne. L'apothéose du poète se déroule sous les regards de pierre de grands auteurs de l'Antiquité, témoins qui cautionnent ce couronnement. De cette assemblée des Anciens, c'est avec Virgile que Racine est le plus souvent comparé, un parallèle particulièrement durable tout au long du XVIII ${ }^{\mathrm{e}}$ siècle. À ce titre, Pierre

20. L'étoile de Racine pâlit dans les années 1820-1830 alors que les romantiques français vont le fustiger, lui, mais surtout ses piètres successeurs et imitateurs, en ce qu'ils incarnent le carcan classique que d'aucuns jugeaient caduque et duquel il fallait absolument se libérer pour l'exploration de nouveaux modèles dramatiques. Sur la critique romantique de Racine, voir Ralph Albanese, Racine à l'école républicaine ou les enjeux socio-politiques de la tragédie classique (1800-1950), Paris, L'Harmattan, 2013, p. 55-57.

21. Stéphane Zékian, Linvention des classiques, Paris, CNRS Éditions, 2012, p. 16. L'auteur, dont l'analyse fine et touffue est consacrée à la «masse critique» hypertrophiée au tournant de 1800 , ajoute, en p. 67, que «la littérature du second $\mathrm{XVII}^{\mathrm{e}}$ siècle divise autant qu'elle fédère ».

22. Ibid., p. 7-24 (Introduction) et p. 38 et suivantes. 
Didot fait de Virgile «le prince des poètes» et de Racine, «le poète français qui a le plus de conformité avec le poète latin ${ }^{23}$ ».

Parangon du classicisme, Racine est élevé au rang de maître de la poésie tragique dont il maîtrise le fond - l'exaltation des passions rendues avec conviction par une fine psychologie - et la forme - le respect absolu des règles et principes classiques et une simplicité, voire un minimalisme dans son discours poétique qui favorise la compréhension intuitive de ses pièces et en renforce la portée didactique ${ }^{24}$. C'est justement pour les vertus de son écriture poétique que les commentateurs des Lumières ont célébré Racine, pour la sensibilité, l'élégance et la simplicité de ses alexandrins, antidotes à la pompe et la grandiloquence de Corneille qui n’a pu en éviter les écueils ${ }^{25}$. Si la censure révolutionnaire a tôt fait d'interdire les textes du poète-courtisan, Napoléon le réinstalle sur le trône des gloires nationales. Doublement canonisé sous l'Empire, à la fois classique culturel et scolaire, il est célébré et enseigné. En 1802, Racine fait partie des 54 écrivains inscrits sur la Liste nationale obligatoire d'auteurs français qui mobilisent les programmes scolaires jusqu'au $\mathrm{xx}^{\mathrm{e}}$ siècle ${ }^{26}$. À n'en point douter, après la Révolution, le tragédien constitue une "valeur de première classe », émule des Shakespeare, Dante, Schiller qui constituent le noble patrimoine littéraire du temps ${ }^{27}$.

Par les Euvres de Jean Racine, Pierre Didot ajoute une pierre au socle identitaire national et supplémente la canonisation du poète. Il se réclame alors de la prestigieuse filiation qui s'enracine dans les classiques latins, s'épanouie avec éclat sous Louis XIV et se prolonge en son temps, dans son édition de 1801 , entre autres expressions cultu-

23. Didot, dans le prospectus du Virgile de 1797, cité par Carol Margot Osborne, op. cit., p. 142.

24. Ralph Albanese, op. cit., p. 17.

25. Depuis les Caractères de La Bruyère (1688), le parallèle entre Racine et Corneille, "couple antithétique indestructible», opposant les passions du cœur à l'exemplarité de l'esprit vertueux, est bien vif, relancé par les commentateurs jusqu'au $\mathrm{XIX}^{\mathrm{e}}$ siècle où il fait encore partie du discours critique et scolaire sous la troisième république. Ibid., p. 20.

26. Daniel Milo, «Les classiques scolaires», Les lieux de mémoire, vol. II, Les nations, Paris, Gallimard, 1997, p. 2085-2086 et p. 2096-2097.

27. François Lagarde, «La valeur et l'effet de nature: réceptions de Racine après la Révolution ", dans Nicholas Cronk et Alain Viala (éd.), La réception de Racine à l'âge classique: de la scène au monument, (SVEC, 2005.08), Oxford, Voltaire Fondation, 2005 , p. 175. 
relles du regain d'intérêt dont jouit Racine. Car le culte du tragédien est protéiforme et inspire autant les écrivains, les imprimeurs que les peintres, les illustrateurs et les gens de théâtre. À cet égard, au début du XIX ${ }^{e}$ siècle, ses pièces sont les plus jouées sur scène en comparaison avec celles de tout autre auteur dramatique ${ }^{28}$. Si Napoléon préfère les «mâles vertus cornéliennes » à la sentimentalité racinienne, se prêtant moins à un «enrôlement patriotique» au service de la propagande impériale, il n'en est pas ainsi du grand public des théâtres ${ }^{29}$. Les tragédies de Racine offrent un éventail de passions dont l'étalage séduit le public de 1800 , lassé de l'héroïsme viril largement mis à profit sous la Révolution. Racine est le poète du cœur. Son théâtre fait sangloter les foules et captive les âmes sensibles devant la sublimité du drame qu'il met en scène.

En matière éditoriale, les textes de Racine font l'objet d'une véritable «panthéonisation de papier ${ }^{30}$ » alors qu'ils sont imprimés et réimprimés aux XVIII et XIX ${ }^{\mathrm{e}}$ siècles, au sein d'éditions pour la plupart modestes et accessibles, destinées aux milieux scolaire et institutionnel, et au réseau des libraires. Pour mieux situer l'apport distinctif des Euvres de Jean Racine dans ce vaste panorama éditorial, je ne m’intéresserai ici qu'aux livres à gravures du XvIII ${ }^{\mathrm{e}}$ siècle. Cohen et Seymour de Ricci en rapportent onze précédant la monumentale édition de 1801, publiés entre 1702 et $1796^{61}$. La majorité de ces livres paraissent en deux ou trois volumes de format in-12 ${ }^{\circ}$, les éditeurs dépassant rarement l'octavo. On y compte 12 figures tout au plus, soit une par pièce, un dispositif visuel somme toute modeste, parfois étoffé d'un frontispice et d'un portrait de Racine. L'édition qui se rapproche le plus de celle de Didot, en matière de format et de grandiloquence, est celle des CEuvres de Racine publiée en 1760 à Paris, en trois volumes in $-4^{\circ}$ agrémentés de 12 figures de l'illustrateur Jacques De Sève. Par contre, la kyrielle de fleurons, culs-de-lampe et vignettes qui en ornent les pages contraste avec le Racine de Didot, saisissant de dépouillement.

28. La Comédie-Française présente près de 90 représentations de Phèdre entre 1801 et 1810 , et plus de 70 d'Andromaque. James Henry Rubin, «Guérin's Painting of Phèdre and the Post-Revolutionary Revival of Racine», The Art Bulletin, 59, décembre 1977, p. 606 (note 29).

29. Stéphane Zékian, op. cit., p. 271-272.

30. Ibid., p. 15.

31. Henri Cohen et Seymour de Ricci, Guide de l'amateur de livres à gravures du XVIII siècle, Paris, A. Rouquette, 1912, p. 844-851. 
À ce titre, l'édition en quatre volumes in-octavo, parue chez Déterville en 1796 , avec 12 figures de Le Barbier, annonce les partis pris esthétiques de l'édition du Louvre. Si elle n'en a pas l'ampleur - on y compte une illustration par pièce tandis que Didot en commande une par acte - elle donne le ton et conjugue sobriété et reconstitution archéologique à l'antique. Toutefois, cette édition et toutes celles qui la précèdent s'enracinent dans la même tradition iconographique d'après le théâtre racinien inaugurée par le dessinateur et graveur François Chauveau (1613-1676). La particularité des vignettes de Chauveau, publiées dans une édition collective de 1676 , tient dans la prédilection de l'illustrateur pour le spectaculaire. Pour plusieurs pièces, il représente ce que l'on ne peut montrer sur scène - duel, empoisonnement, meurtre, etc. - et que Racine a choisi de décrire par la narration, dans le respect des règles de la bienséance classique formulées par Boileau: «ce qu'on ne doit point voir, qu'un récit nous l'expose ${ }^{32}$ ». Pierre Didot est le premier à s'affranchir de cette tradition illustrative pour mieux renouer avec le décorum du Grand siècle et respecter la conception théâtrale du tragédien canonique. Les cinquante-six compositions du Racine de 1801 se veulent au plus près de l'esthétique classique française. À contre-courant des traditions du livre et de l'illustration qui prévalaient depuis plus d'un siècle, l'édition nationale de Pierre Didot pérennise l'esthétique d’une époque révérée par les élites cultivées de la France du Directoire et de l'Empire.

\section{Le monument politique}

Un autre protagoniste s'insinue dans cet Élysée des grands hommes incarné dans les Euvres de Jean Racine: Napoléon Bonaparte (17691821). Le Consul, bientôt Empereur, contribue à la frénésie du souvenir qui caractérise son temps et souscrit à l'idéalisation du siècle de Louis XIV à condition que celui-ci ne soit pas plus grand que «le bel aujourd'hui du siècle de Napoléon ${ }^{33}$ ». L'Empereur a cherché son règne durant à le légitimer par l'histoire ancienne, en se présentant comme l'héritier de Charlemagne ou de Jules César, et surtout par le passé monarchique français: si on doit le comparer, il préfère par-dessus tout

32. Alain-Marie Bassy, op. cit., p. 158.

33. L'éloquente formule est de Stéphane Zékian, op. cit., p. 32. 


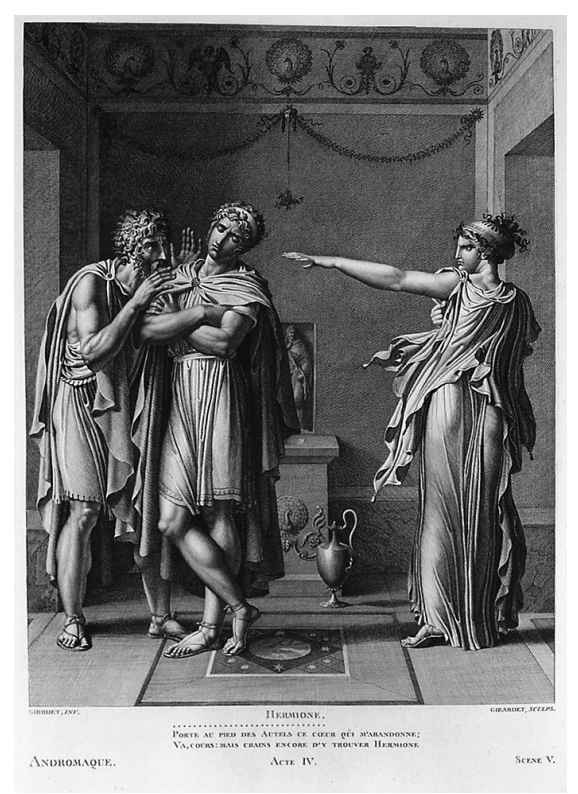

Figure 1. Euvres de Jean Racine, tome 1 Paris : Didot, 1801

Andromaque, acte $I V$, scène v 1792-1801 / Gravure en taille douce $26 \times 20 \mathrm{~cm} /$ sur papier

Dessiné par Girodet / Gravé par Girardet

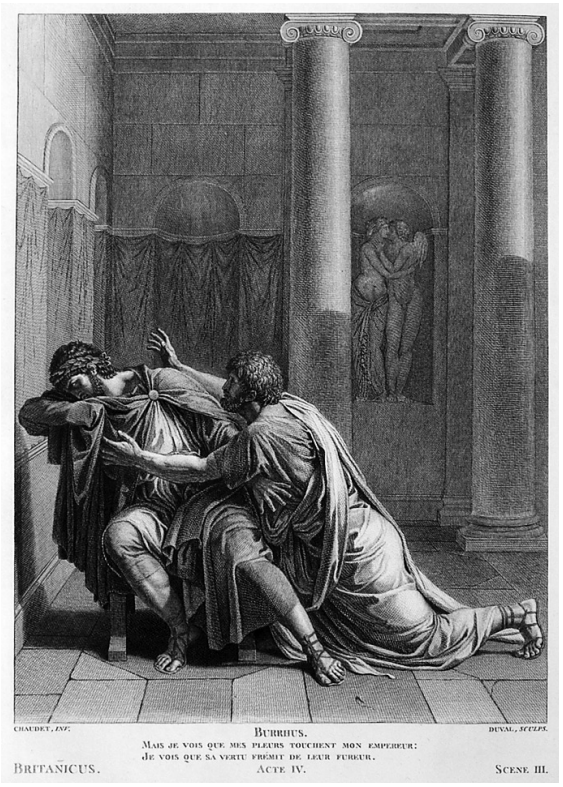

Figure 2. Euvres de Jean Racine, tome 1 Paris : Didot, 1801

Britannicus, acte IV, scène iii 1792-1801 / Gravure en taille douce $26 \times 19.5 \mathrm{~cm} /$ sur papier

Dessiné par Chaudet / Gravé par Duval

Les images sont reproduites avec l'aimable autorisation de la Bibliothèque des livres rares et collections spéciales de l'Université de Montréal.

Elles sont tirées de l'exemplaire 105 de 250 des (Euvres de Jean Racine, Paris, Imprimerie de P. Didot l'Aîné, 18o1, 3 volumes in-folio.

que ce soit avec Louis XIV ${ }^{34}$. Pour Napoléon, le pouvoir absolu est le seul cadre au sein duquel peut fleurir l'excellence littéraire et artistique. Il lui arrive «de se rêver en Louis le Grand et d'appeler de ses vœux un nouveau Racine, un nouveau Corneille, pour le célébrer ${ }^{35}$ »: la propagande impériale conditionne d'ailleurs un mécénat à la manière du Roi-Soleil pour encourager la création au service du régime.

34. Idem.

35. Catriona Seth, «L'Institut et les prix littéraires», dans Jean-Claude Bonnet (éd.), L'empire des Muses. Napoléon, les arts et les lettres, Paris, Belin, 2004, p. 121. 


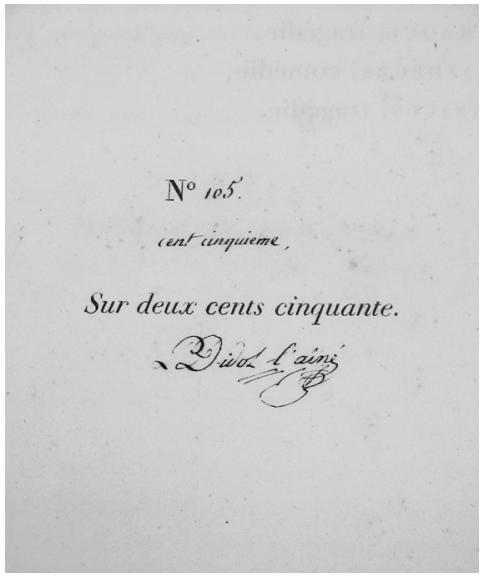

Figure 3. Euvres de Jean Racine, fin du tome 1

Paris : Didot, 1801

Numérotage et signature manuscrite de Pierre Didot l'Aîné

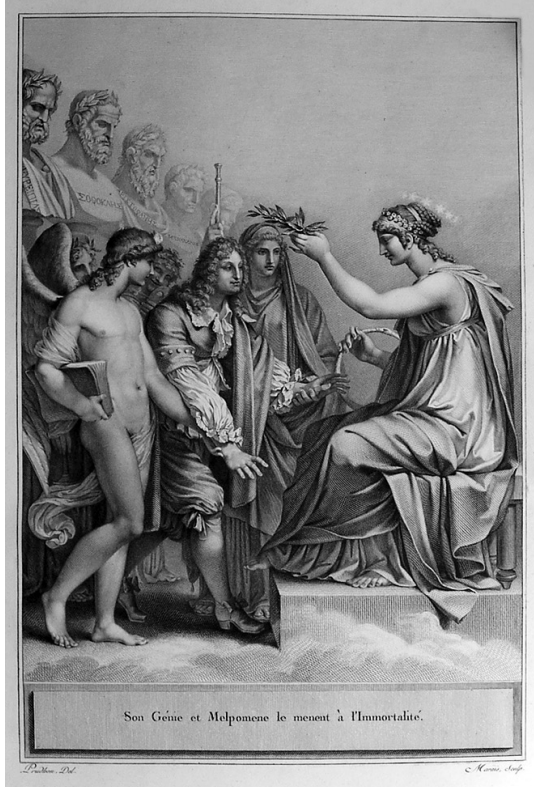

Figure 4. Euvres de Jean Racine, tome 1 Paris: Didot, 1801

Frontispice: Portrait de Racine 1792-1801 / Gravure en taille douce $30 \times 21 \mathrm{~cm} /$ sur papier

Dessiné par Prud'hon / Gravé par Marais

On pourrait croire que Pierre Didot connaissait avant l'heure les inclinations du futur Empereur lorsqu'il a décidé de lui dédier sa prestigieuse édition nationale. Sa dédicace renforce d'une troisième dimension, politique, la conception monumentale du Racine en ce que le livre exalte la gloire du Premier Consul et du nouveau régime. Sur un ton poétique et flagorneur, l'éditeur célèbre l'œuvre " pacificatrice» du «favori de Mars» - le contexte étant celui, précaire, de la paix signée avec l'Autriche et scellée, grâce au Consul, par le Traité de Lunéville de $18 \mathrm{o}^{36}$. Une prière s'insinue dans ses vers: «Oui, préside aux travaux, anime l'industrie / Fais d'un nouvel éclat rayonner ma patrie $^{37} »$. Didot espère s'attirer les faveurs de Napoléon dans une

36. Pierre Didot l’Aîné, «Au Général Bonaparte, Premier Consul de la République française », Euvres de Jean Racine, op. cit., t. I, p. 1 de la dédicace.

37. Idem. 
société qui renoue lentement, après les secousses révolutionnaires, avec un important financement des arts par l'État concrétisé sous l'Empire. Fierté de l'industrie nationale et réminiscence d'une époque dorée de la tradition artistique et littéraire française, le livre-monument est envisagé comme une opération de charme destinée au pouvoir. À l'héroïsme du tragédien et des ouvriers du livre, s'ajoute celui de Napoléon Bonaparte, par un discours qui complémente les textes de Racine. La dédicace et la préface sont d’ailleurs parsemées d'expressions tirées du lexique de la gloire et de l'héroïsme: «lauriers », «victoires », «exploits», «nobles» et autres vocables similaires ponctuent les phrases de ces textes préliminaires porteurs de valeurs morales. Les illustrations leur font écho et se déclinent comme un catalogue de vertus amplifiant la portée idéologique de l'ouvrage. Des valeurs donc qui sont celles du régime politique que le livre honore et de l'institution de laquelle il est issu. Faisant partie de la collection sélecte des éditions du Louvre, le Racine est rattaché à ce lieu de pouvoir d'où Didot gère ses presses, grâce au Consul. Le livre-monument est œuvre d'art institutionnelle, un art officiel en parfaite adéquation avec le modèle intellectuel et l'idéologie politique de la société qui le berce.

\section{Le contexte de réception des Euvres de Jean Racine}

La fortune-critique de l'édition de 1801 appuie plus encore sa désignation comme un art officiel, encensé par les jurys des grandes expositions. Le majestueux livre remporte la médaille d'or lors de la deuxième exposition publique des produits de l'industrie française de 1801. Il est alors estimé, avec l'Horace de 1799, comme "plus belle[s] production[s] de la typographie de tous les pays et de tous les âges », un jugement confirmé par la première exposition universelle de Londres en $1851^{38}$. Il s'agit là du seul fait saillant de la fortune-critique du Racine. Ce jugement somme toute flatteur repose sur la matérialité même du livre évalué à l'aune de sa perfection typographique, laquelle étant seule digne de passer à l'histoire. Il faut dire que la stratégie éditoriale qui sous-tend l'ouvrage conditionne son prestige et sa considération tel un chef-d'œuvre du grand art dans une société qui valorise la mémoire

38. Pour le jugement complet tiré du Rapport de l'exposition (13 ventôse An IX), voir Carol Margot Osborne, op. cit., p. 177, note 24. 
des grands hommes et les réalisations artistiques d'envergure, voire propagandistes. Pourtant, l'ouvrage ne rencontre pas son public.

Après la Révolution, les publics de l'art constituent un ensemble particulièrement bigarré, constitué d'anciennes noblesses françaises, bientôt aristocraties d'Empire, de "parvenus » ou nouveaux riches qui ont fait fortune grâce aux révolutions française et industrielle, et d'une petite bourgeoisie commerçante. Au sein de cette masse critique en ébullition, Pierre Didot envisage de séduire le plus grand nombre, par l'entremise du livre, a priori démocratique dans une France qui s'alphabétise avec l'avancée du XIXe siècle. Dans une Épitre sur la nécessité d'encourager les artistes, présentée à l'Institut National en 1799, l'éditeur reconnaît que «les beaux-arts sont pour tous» et souhaite que «l'œil s'exerce ${ }^{39}$ ». Ses entreprises éditoriales semblaient idéales pour éduquer le public au beau et diffuser le grand goût, notamment pour les tragédies classiques et la peinture d'histoire, auprès des différentes classes de la société du temps. Pourtant, la stratégie éditoriale du Racine (format, numérotage, signature manuscrite, illustrations rivalisant avec la peinture d'histoire, etc.) et son prix exorbitant ${ }^{40}$ démontrent qu'il était tout sauf un ouvrage de diffusion populaire. D'autant plus que le culte de Racine, en 1800 , n'est pas célébré par le commun des mortels. Après la Révolution, le tragédien demeure méconnu de la plupart des Français. Encore fallait-il avoir reçu une éducation convenable pour en faire la rencontre à l'école, Racine canonique, tel un passage obligé scolaire et culturel, et cultiver une finesse d'esprit qui permette d'en apprécier les alexandrins et d'en saisir les délicatesses. Jean Racine brille avant tout comme celui qui fait «les délices des hommes instruits» tels que les décrit La Harpe, et qui, peut-être comme lui, sont nostalgiques des valeurs de l'Ancien Régime ${ }^{41}$. Le tragédien est le fétiche des connaisseurs raffinés, engagés dans les univers politique, académique ou culturel et principal public des Salons et des théâtres. Les Euvres de Jean Racine semblaient tout

39. Didot cité par Osborne, op. cit., p. 110.

40. Achetés par souscription, les trois volumes coûtaient 1200 francs à celui qui les commandait à la parution du premier volume et 1800 francs à celui qui attendait au troisième. Des sommes gonflées à 1800 et 2700 francs pour les 100 exemplaires plus luxueux avec épreuves avant la lettre.

41. Jean-François de La Harpe, Éloge de Racine, par M. de La Harpe, Amsterdam, Lacombe, 1772 , p. 5 ०. 
destinées à une élite intellectuelle et financière dans le giron de Napoléon.

L'identité précise des souscripteurs du Racine n'est pas connue. L'historien et bibliographe Paul Lacroix (1806-1884) a recueilli le témoignage d'un ami de Pierre Didot, le Baron Walckenaer (1771-1852), qu'il rapporte dans la Notice historique sur l'Édition de Racine, dite du Louvre et sur ses estampes, datée du $1^{\text {er }}$ novembre $1876^{42}$. Walckenaer donne quelques indices sur l'identité sociale des présumés souscripteurs: quelques membres du Sénat conservateur, des militaires dont certains généraux, des fournisseurs aux armées, des banquiers. L'historienne Carol Margot Osborne rapporte qu'outre le sceau de l'Empereur, on retrouve le cachet de Joseph Bonaparte sur quelques exemplaires du Racine, de même qu'on peut supposer que le Général Junot et le Duc d'Abrantès l'aient acquis puisqu'ils étaient reconnus comme de véritables bibliophiles et collectionneurs ${ }^{43}$. Talleyrand, ministre des relations extérieures sous le Consulat, prévoyait l'acquisition de douze copies des Euvres de Jean Racine, comme le confirme une lettre du ministre de $1798^{44}$. Outre ces quelques prestigieux collectionneurs, et selon l'aveu du Baron Walckenaer, plusieurs acheteurs se seraient déclarés et auraient souscrit à l'édition pour entrer dans les bonnes grâces du Premier Consul qui venait d'en accepter la dédicace. À la lumière de ce témoignage, on peut se demander si l'ouvrage n'aurait pas servi de «savonnette à vilain ${ }^{45}$ » culturelle, soit une œuvre prestigieuse que l'on acquiert pour se donner un lustre duquel on est dépourvu. Telles ces charges que l'on pouvait acheter pour s'ennoblir, le Racine s'impose au bon goût à une époque où le patrimoine aristocratique est endossé par un groupe social désireux de se montrer

42. Paul Louis Jacob (Lacroix se faisait appeler Bibliophile Jacob), Notice historique sur l'Édition de Racine, dite du Louvre et sur ses estampes, $1^{\mathrm{er}}$ novembre 1876 , non paginée. Ce document a été inséré dans l'exemplaire 198 de 250 des (Euvres de Jean Racine conservé à l'Arsenal (Bibliothèque de l'Arsenal, GR FOL 122 1;2;3), probablement par son auteur lui-même, ancien conservateur à l'Arsenal. La notice de 1876 a été publiée un an plus tard dans le grand folio Collection des cinquante-sept estampes dessinées et gravées pour les ouvres de J. Racine, édition du Louvre, par les premiers artistes de la République française. Avec une notice historique par P. L. Jacob bibliophile, Paris, L. Willem, 1877.

43. Anthony Griffiths, op. cit., p. 143 et Carol Margot Osborne, op. cit., p. 99-101.

44. Ibid., p. 173-174, note 36.

45. La savonnette à vilain est le nom donné aux procédés de passage de la roture à la noblesse (Littré). 
savant. Sans en goûter le plaisir délicat, la nouvelle bourgeoisie cultive Racine par souci de distinction et par mimétisme d'une classe sociale estimée pour son raffinement et dont le pouvoir culturel est encore vif en 1800 . Ce public potentiel considérait de surcroît la bibliothèque familiale comme l'incarnation vivante de la culture, transmettant l'identité sociale de la famille et ses valeurs ${ }^{46}$. Dans ce contexte, il était bien vu de la garnir d'œuvres distinctives. Le Racine aurait alors profité d'un éphémère engouement auprès de ces mystérieux souscripteurs qui, à défaut d'être de véritables collectionneurs, ont saisi l'occasion de s'approprier un livre d'exception qui conférait une légitimité intellectuelle à leur collection.

Peu de souscripteurs auraient acquis l'ensemble complet des Euvres de Jean Racine en trois volumes, plusieurs copies ont d'ailleurs été démantelées et dispersées. Pierre Didot a conservé 98 copies invendues jusqu'à sa mort, en $1853^{47}$. Ambroise-Firmin Didot témoigne qu'en 1855 , près de 160 exemplaires sur 250 croupissent toujours sur les tablettes des libraires ${ }^{48}$. Les autres éditions du Louvre n'ont guère connu un meilleur sort. L'inventaire après-décès de Pierre Didot révèle qu'il possédait toujours 50 copies du Virgile, 42 des Fables et 61 de l'Horace $^{49}$. Malgré leur caractère fragmentaire, ces données témoignent de la difficulté à écouler l'entièreté de ces tirages exclusifs. Cette série marque d'ailleurs la fin d'une époque dorée dans la carrière de Pierre Didot. Les Euvres de Jean Racine lui auraient coûté 2 millions de francs et l'ont contraint à vendre sa maison ${ }^{50}$. En 1804, l'Empereur le met à la porte du Palais pour cause de réaménagement. Choqué, Didot condamne les «dix années stériles» de l'Empire, témoins de l'essoufflement de sa production ${ }^{51}$.

Les Euvres de Jean Racine connaissent certes un succès d'estime lors de deux expositions de prestige, mais ces éloges officiels ne peuvent

46. Alain-Marie Bassy, op. cit., p. 145 et Jean Viardot, «Livres rares et pratiques bibliophiliques », dans Henri-Jean Martin et Roger Chartier (éd.), op. cit., p. $44^{8}$.

47. D'après l'inventaire après-décès. Carol Margot Osborne, op. cit., p. 101 et 135.

48. André Jamme, Les Didot: trois siècles de typographie et de bibliophilie, 1698 1998, Paris, Agence culturelle de Paris, 1998, p. 46.

49. Carol Margot Osborne, op. cit., p. 101.

50. Ibid., p. 88 et Anthony Griffiths, op. cit., p. 145.

51. Didot cité par Anthony Griffiths, ibid., p. 146. 
masquer le silence assourdissant des commentateurs du temps $s^{52}$. La reconnaissance du livre-monument à l'exposition des produits de l'industrie de 1801 aurait certainement dû susciter les réactions critiques, tout comme la présentation de certaines planches et dessins originaux au Salon, la réédition de l'ouvrage en format in-octavo en 1816, en dépit de la reprise des gravures par des artistes secondaires, et la réimpression entre 1807 et 1812 des gravures originales par Charles Landon dans ses Annales du musée et de l'École moderne des beaux-arts ${ }^{53}$. Pourtant, dans les journaux et périodiques du temps, on ne retrouve aucune mention du Racine, autre infortune du projet de Didot. Il faut attendre la fin du $\mathrm{XIX}^{\mathrm{e}}$ siècle et la critique de Paul Lacroix pour voir le Racine encensé dans sa notice de $1876^{54}$. Celui-ci y va d'ailleurs de son hypothèse quant au silence de la presse et explique que «ce magnifique livre restait inabordable à la critique » en ce que tous les exemplaires étaient passés «directement chez les souscripteurs ${ }^{55}$ ». C'est là omettre l'influence de la médaille d'or de 1801 , la réédition de l'ouvrage ou la réimpression des planches, et donc omettre «la vie du livre» en dehors des ventes par souscription, particulièrement maigres si l'on considère les invendus rapportés dans les années 1850 .

\section{Les infortunes: quelques pistes d'interprétation}

L'ambitieux projet éditorial de Pierre Didot échoue dans la durée, subtilement. Chef-d'œuvre typographique acclamé en 1801 et 1851, hommage à Racine dont il préserve la mémoire et célèbre le talent dans un contexte de culte frénétique des ancêtres nationaux, objet de collection à l'image d'un régime politique qu'il exalte et sert, les

52. Paul Lacroix constate ce mutisme, en 1877 : «On peut dire que l'édition complète n'avait jamais été connue, car la critique d'art ne s'en était pas occupée à l'époque de la publication, et nous n'avons pu découvrir, en feuilletant les journaux et les revues littéraires ou artistiques, de ce temps-là, un seul article, un seul compterendu relatif à ce magnifique livre, qui méritait tant d'éloges et qui n'en eût pas dans la presse». Paul Lacroix, op. cit., p. 17.

53. Landon a publié 82 gravures au trait issues des éditions du Louvre de Didot. Carol Margot Osborne, op. cit., p. 100 et p. 174, note 37. Notons également que le libraire Charles Garnier a reproduit 13 planches du Racine dans une édition in-octavo des Euvres complètes de Racine. La première publication date de 1853 et des rééditions suivront en 1858, 1861, 1864 et 1867. Voir A. J. Pons, op. cit., p. 45 et suivantes.

54. Ralph Albanese, op. cit., p. 87.

55. Paul Louis Jacob, op. cit., p. 17. 
Euvres de Jean Racine annonçaient un succès qui ne s'est jamais concrétisé. Ses contextes de production et de réception sont dissonants. Fondées sur une surenchère de cette monumentalité décortiquée dans ces pages, et en dépit d'une stratégie éditoriale que d'aucuns pourraient estimer extravagante dans les années 1790, le livre est reçu dans l'indifférence. Pour conclure, attardons-nous à trois des hypothèses les plus signifiantes venant éclairer cette réception mitigée.

Le libraire et collectionneur Antoine-Augustin Renouard (17651853) constate en 1819 que les « in-folios sont aussi peu goûtés du public qu'ils lui sont peu utiles ${ }^{56}$ ». Déjà au XviII ${ }^{\mathrm{e}}$ siècle, nous l'avons vu, le format in-folio est une rareté, encore plus au XIX ${ }^{\mathrm{e}}$ siècle. Les éditions du Louvre, parmi d'autres ouvrages d'envergure publiés dans les mêmes années, renouent avec la grandiloquence du livre du XVII siècle et constituent une vogue bien éphémère. Comme l'affirme l'historien Jean Viardot, «la bibliophilie Révolution-Empire crispée sur le domaine des Belles-Lettres classiques antiques et modernes et engouée de petites sophistications quant aux exemplaires, s'insère un peu comme une parenthèse entre le XvirI et la nouvelle bibliophilie qui va naître vers $1830^{57}$ ». Cet important changement de paradigme marque le passage de l'imprimerie de Gutenberg à l'ère industrielle en même temps que grimpe l'alphabétisation et que se développent les technologies d'impression ${ }^{58}$. Avec elles, de nouvelles techniques d'estampe - lithographie, pointillé, acier - moins coûteuses et fastidieuses, remplacent les anciennes, telle la gravure sur cuivre pour laquelle Didot a tant investi pour s'assurer de la qualité de ses éditions du Louvre. Le Racine se tient donc à l'aube d'une révolution livresque qui a certainement influencé l'accueil qu'on lui a réservé. Impossible à lire, l'ouvrage peut tout juste être exposé, présenté comme un objet précieux dont on exhibe la magnificence. Il est de fait moins livre à lire, qu'objet de collection d'une bibliophilie de luxe qui semble archaïque dès les premières décennies transitoires du XIX ${ }^{e}$ siècle. En revanche, si l'édition est en lente mutation, le milieu des bibliothèques est véritablement en crise dans la France révolutionnée. La législation de la Révolution, relative aux ordres monastiques et à l'émigration, a causé

56. Renouard, dans son Catalogue de la Bibliothèque d'un Amateur de 1819, cité par Anthony Griffiths, op. cit., p. 146.

57. Jean Viardot, op. cit., p. 448.

58. Frédéric Barbier et David Bellos, op. cit., p. 542-543. 
la perte d'un grand nombre de collections et de bibliothèques ${ }^{59}$. Compte tenu de la canonisation culturelle et scolaire de Racine, on peut supposer que l'édition de Didot était destinée à ces réseaux de bibliothèques scolaires ou religieuses qui n'étaient pas en mesure d'en faire l'acquisition durant cette époque en rupture et en redéfinition.

Il faut en outre considérer le statut de l'illustration gravée en 1800 comme un facteur décisif ayant teinté la réception du Racine. Dans un extrait du Journal du Bulletin universel des sciences, des lettres et des arts de 1799, le dédain pour les entreprises alliant art et commerce est manifeste: «la gravure étant devenue aujourd'hui une entreprise plutôt qu'un art, nous n'en dirons qu'un mot... »; ou encore, dans une édition du Mercure de France de 1798, Chaussard affirme sur le même ton que «dans les Arts, dès que l'intérêt du gain approche, le génie s'éloigne ${ }^{60} »$. La faute est impartie à l'illustration, encore considérée comme un sous-métier en 1800 , et à la lucrative gravure, et ce, malgré la revalorisation du burin par Napoléon qui inaugure en 1802 un prix de Rome réservé à cette seule technique. Pourtant, pour les Italiens, comme pour les Anglais, les entreprises éditoriales d'envergure étaient considérées comme "une branche de commerce des plus glorieuses » selon les mots de Didot dans une lettre de 1797 adressée au ministre de l'intérieur, François Neufchâteau ${ }^{61}$. L'éditeur espérait qu'il en soit ainsi en France puisqu'il y voyait un excellent moyen de stimuler le marché de l'art et de soutenir les artistes en mal de commandes. À cet égard, il ajoute la même année: «on serait étonné de la quantité d'Artistes et d'ouvriers que peut occuper ou alimenter l'art de l'imprimerie $^{62} »$. Plusieurs artistes et critiques français rechignaient cependant encore à envisager l'art par le prisme du commerce, qui ne pouvait que l'avilir. David partageait sans doute cette opinion car malgré sa longue collaboration avec Didot pour les éditions du Louvre, son nom ne figure nulle part au sein des livres pas plus que ses activités d'illustrateur ne sont commentées au Salon ${ }^{63}$. Les gravures et dessins originaux

59. Louis Trenard "Introduction. De l'histoire des bibliothèques", Les bibliothèques au XVIII siècle, sous la dir. de Louis Trenard, Bordeaux, Société des bibliophiles de Guyenne, 1989, p. 53.

6o. Les deux extraits sont rapportés par Carol Margot Osborne, op. cit., p. 97.

61. Didot cité par Carol Margot Osborne, ibid., p. 48-50.

62. Didot cité par Philippe Kaenel, Le métier d'illustrateur 1830-188o. Rodolphe Töpffer, J.-J. Grandville, Gustave Doré, Paris, Messene, 1996, p. 27.

63. Carol Margot Osborne, op. cit., p. 84-85. 
des éditions du Louvre ont d'ailleurs été pour la plupart ignorés des commentateurs des expositions. Enfin, ce climat particulier explique peut-être pourquoi les copies sur vélin du Virgile et du Racine, avec dessins originaux, ont été mises aux enchères par leur propriétaire Firmin Didot en 1810 sans même trouver preneur ${ }^{64}$. La déconsidération de l'illustration et de la gravure, manifestement boudées en France autour de 1800 , jette une lumière nouvelle sur les infortunes des Euvres de Jean Racine et sur le silence des critiques du temps.

Une dernière piste de réflexion contextuelle s'impose enfin. En France, le milieu de l'imprimerie de 1789 à 1830 est surtout influencé par la conjoncture politique ${ }^{65}$. Les incessants changements provoqués par l'alternance des régimes, de la Révolution au Directoire puis du Consulat à l'Empire, ont certainement troublé le commerce du livre, mais surtout l'accroissement du public potentiel du Racine. Les liseurs fortunés qui ont contribué aux premiers succès des Didot dans les années 1780 ont pour la plupart été guillotinés, sont morts au combat durant les guerres révolutionnaires ou impériales, ou ont été ruinés. Le public destiné à admirer et à chérir le livre-monument s'est évanoui avec la Révolution, et bientôt avec la chute de l'Empire. Le Bonaparte de la dédicace n'est plus. Les collectionneurs nostalgiques de l'Ancien Régime sont en voie de disparition. Et le livre croupit sur les étagères des libraires. Le monument semble dès lors voué à la ruine de concert avec la disparition de ceux qui devaient en assurer la postérité. C'est dire qu'une œuvre qui s'imprègne d'un régime politique, qui s'en fait la représentation officielle, tant dans sa forme que dans son idéologie, risque le même sort que ledit régime, notamment dans la France du XIX ${ }^{e}$ siècle où l'éphémère caractérise le politique.

64. Ibid., p. 100 et Henri Cohen et Seymour de Ricci, op. cit., p. 850.

65. Frédéric Barbier et David Bellos, op. cit., p. $55^{2}$. 